\title{
PROGRESS AND OPEN PROBLEMS IN HYPERNUCLEAR STRUCTURE
}

\author{
BNL -45238 \\ D. J. Millener \\ Physics Department \\ DE91 001114 \\ Brookhaven National Laboratory \\ Upton, NY 11973
}

\begin{abstract}
$\Lambda$ hypernuclei exist as particle-stable systems with spectroscopies analogous to those of ordinary nuclei. The analysis of Brookhaven data on $\left(\pi^{+}, K^{+}\right)$reactions, for selected targets up to $A \sim 90$, has yielded information on $\Lambda$ single-particle energies and thus the $\Lambda$-nucleus mean field. Better energy resolution and higher intensity beams are needed to carry out coincidence experiments and detailed spectroscopic studies. The prospects for investigating hypernuclear structure at high-energy electron accelerator farilities via the $\left(e, e^{\prime} K\right)$ or $(\gamma, K)$ reactions, which are kinematically similar to the $\left(\pi^{+}, K^{+}\right)$reaction, but which favor spin-flip transitions, are also considered.
\end{abstract}

Invited talk presented at

PANIC XIIth, International Conference on Particles and Nuclei MIT, Cambridge, MA - 25-29 June 1990

This manuscript has been authored under contract number DE-AC02-76CH00016 with the U.S. Department of Energy. Accordingly, the U.S. Government retains a non-exclusive, royalty-free license to publish or reproduce the published form of this contribution, or allow others to do so, for U.S. Government purposes.

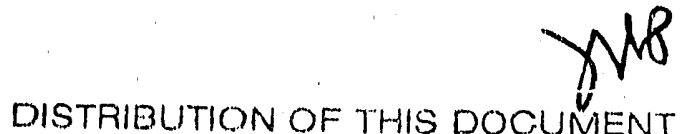




\title{
PROGRESS AND OPEN PROBLEMS IN HYPERNUCLEAR STRUCTURE
}

\author{
D. J. Millener
}

Physics Department, Brookhaven National Laboratory, Unton, N. Y. 11973

$\Lambda$ hypernuclei exist as particle-stable systems with spectroscopies analogous to those of ordinary nuclei. The analysis of Brookhaven data on $\left(\pi^{+}, \mathrm{K}^{+}\right)$reactions, for selected targets up to $A \sim 90$, has yielded information on $\Lambda$ single-particle energies and thus the $\Lambda$-nucleus mean field. Better energy resolution and higher intensity beams are needed to carry out coincidence experiments and detailed spectroscopic studies. The prospects for investigating hypernuclear structure at high-energy electron accelerator faciitities via the $\left(e, e^{\prime} \mathrm{K}\right)$ or $(\gamma, \mathrm{K})$ reactions, which are kinernatically similar to the $\left(\pi^{+}, \mathrm{K}^{+}\right)$reaction, but which favor spin-flip transitions, are also considered.

Since a single $\Lambda$ behaves as a distinguishable particle in the nucleus, it car occupy any single-particle orbit in the $\Lambda$-nucleus average field. Well-defined series of peaks corresponding to nodeless single-particie states have been identified in heavy nuclei using the $\left(\pi^{+}, \mathrm{K}^{+}\right)$ reaction at Brookhaven ${ }^{1}$. The spacings of the $\Lambda$ single-particle levels for a given $A$ constrain both the radius and the depth of the $\Lambda$ potential well. A Woods-Saxon well with a depth of $28 \mathrm{MeV}$; a diffuseness of $0.54 \mathrm{fm}$ and radius parameter $r_{0}=1.128+0.439 A^{-2 / 3} \mathrm{fm}$ (compared with $1.144-1.276 A^{-2 / 3}$ for the charge density) gives an excellent fit to the data (see Fig. 1 of Ref. 2), as do phenomenological $\Lambda$-nucleus potentials ${ }^{2,3}$, based on a spherical Skyrme Hartree-Fock approach. The essential features of the potentials are an attractive component linear in the nuclear density (taken proportional to the empirical charge density) with a depth of $\sim 60 \mathrm{MeV}$ (consistent with that obtained by folding the free $\Lambda \mathrm{N}$ interaction with the density), a repulsive component proportional to a higher power of the density (giving the potential a radius larger than that of the underlying density) and a non-locality (through an effective mass of $\sim 0.8 m_{\Lambda}$ in the nuclear interior), which gives an energy dependence to the equivalent local potential, to fine tune the fit to binding energies over the full mass range.

The Brookhaven data ${ }^{1}$ has inspired a number of theoretical descriptions of the spectra, the most complete calculations being those of Motoba et al. ${ }^{4}$. Recentily, a full analysis of the experimental data has been completed and absolute cross sections are available (R. Chrien, preceding talk). For example, in the case of ${ }^{89} \mathrm{Y}$, shown in Fig. 1, the major contributions to the peaks arise frc:n high-spin couplings of $l_{\Lambda} g_{9 / 2}^{-1}$ configurations (dashed curves). Since the spin-dependent components of the $\Lambda \mathrm{N}$ effective interaction are small, ${ }^{6}$ the fine structure of the peaks is not resolvable with the typical experimental energy resolution ${ }^{1}$ of $3 \mathrm{MeV}$. The

This manuscript has been authored under contract number DE-AC02-76CH00010 with the U.S. Department of Energy. 
clear peak structure exhibited in Fig. 1; even with this resolution, for the ${ }^{89} \mathrm{Y}\left(\pi^{+}, \mathrm{K}^{+}\right){ }_{\Lambda}^{89} \mathrm{Y}$ reaction results from the interplay of a number of factors, including the nature of the neutron orbitals near the Fermi surface, the high momentum transfer and the role of distortion. Neutron pickup reactions ${ }^{5}$ on ${ }^{90} \mathrm{Zr}$ show that the $1 g_{9 / 2}$ strength is concentrated in the ${ }^{89} \mathrm{Zr}$ ground state, with appreciable fractions (about one half) of the $2 p_{1 / 2}, 2 p_{3 / 2}$ and $1 f_{5 / 2}$ strength in low-lying states, The remaining strength associated with $f p$ orbits is highly fragmented with, for example, the $1 f_{7 / 2}$ strength so far located being spread from $4-21 \mathrm{MeV}$ in excitation energy with a centroid at $\sim 10.3 \mathrm{MeV}$. The peaks in Fig. 1 are mainly due to the conversion of a $1 g_{9 / 2}$ neutron into a $\Lambda$ in the orbit specified (dashed'curves), where the notation $l_{\Lambda}$ inchudes both spin-orbit partners. The high spin of the last-filled, $g_{9 / 2}$ orbit provides many neutrons (10, to which the pickup data are normalized, in the simple shell-model limit) on which the conversion can take place and enables the preference for high momentum and angular momentum transfer to be satisfied. The strength associated with the conversion of $f p$ neutrons into a $\Lambda$ is less than that for $g_{9 / 2}$ neutrons and is widely spread, but, as can be seen from Fig. 1, makes a significant contribution beneath the $f_{\Lambda}$ and $g_{\Lambda}$ peaks.

Table 1: Theoretical cross sections $(\mu b / s r)$ for the ${ }^{90} \mathrm{Zr}\left(\pi^{+}, \mathrm{K}^{+}\right){ }_{\Lambda}^{90} \mathrm{Zr}$ reaction.

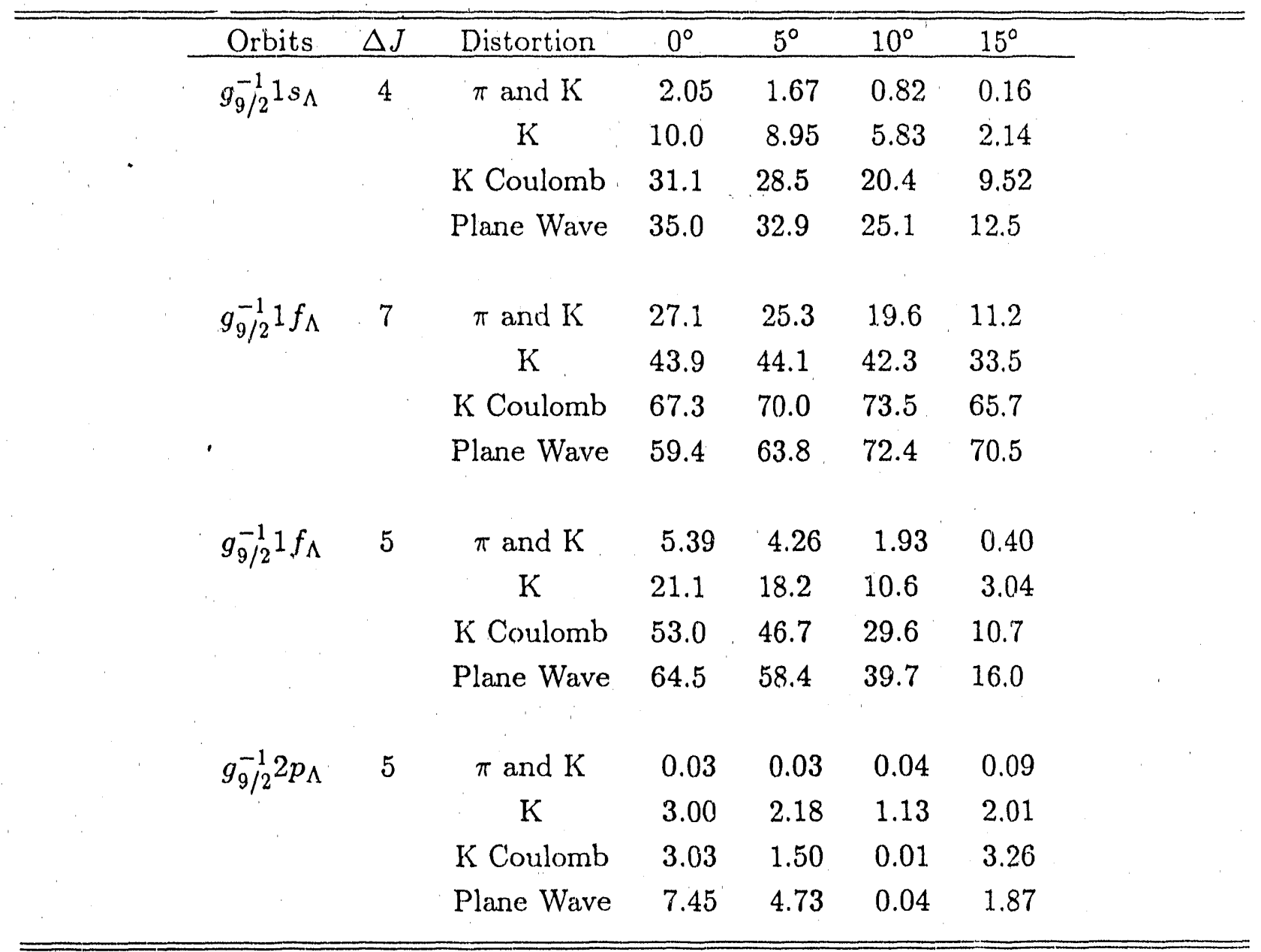




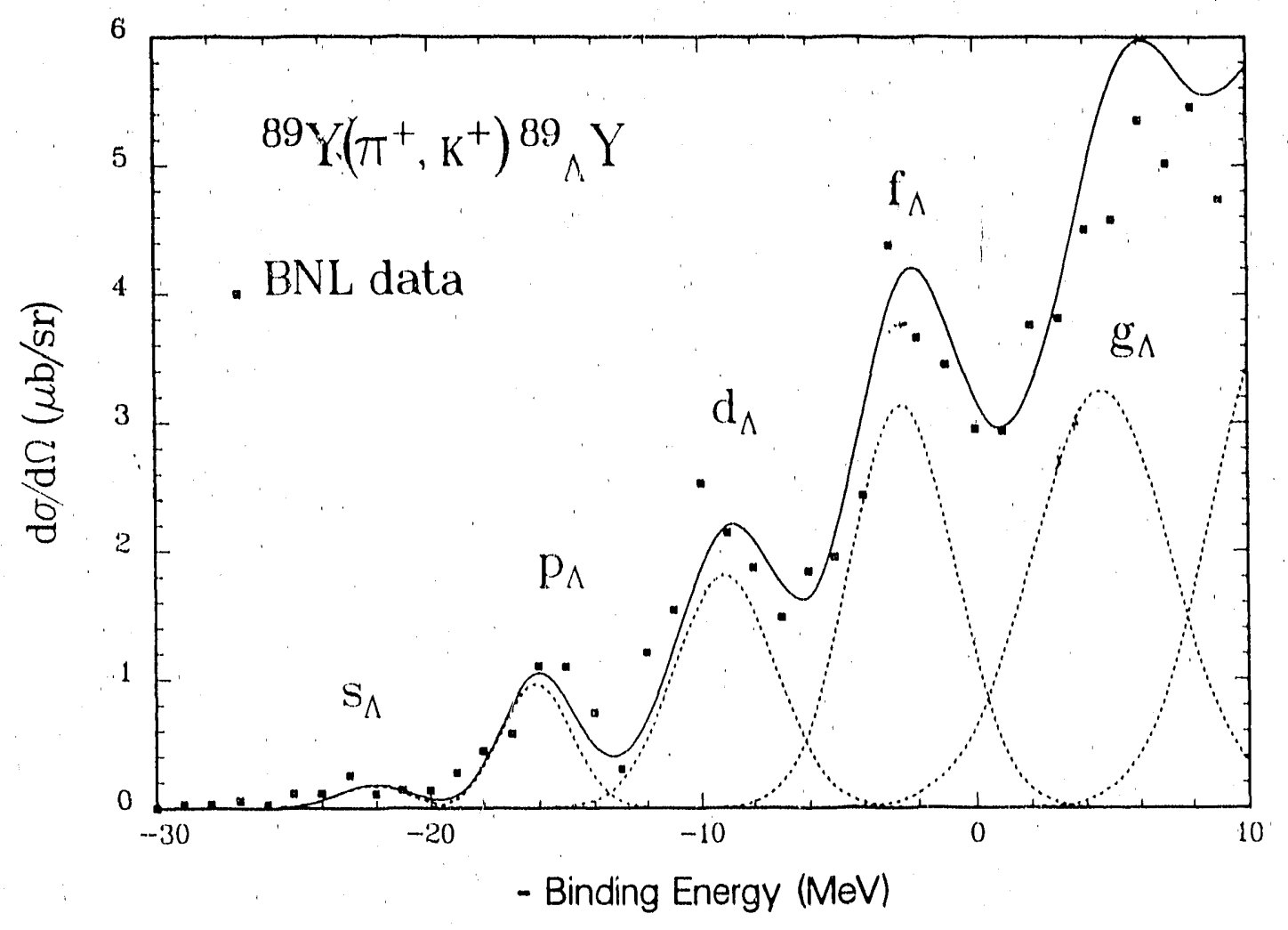

Figure 1: The excitation spectrum for the ${ }^{89} \mathrm{Y}\left(\pi^{+}, \mathrm{K}^{+}\right){ }^{89} \mathrm{Y}$ reaction at $1.05 \mathrm{GeV} / c$ and $\theta_{K}=10^{\circ}$, from an AGS experiment, ${ }^{1}$ The curves represent theoretical calculations (multiplied by an overall factor of 0.71 ) which use a.Fermi-averaged $0^{\circ}$ elementary amplitude, optical potentials fit to elastic scattering at $800 \mathrm{MeV} / \mathrm{c}$, Woods-Saxon bound state potentials, and the experimental distribution of neutron pickup strength ${ }^{5}$ from ${ }^{90} \mathrm{Zr}$ (normalized to 10 for the $g_{9 / 2}$ orbit). The $l_{\Lambda} g_{9 / 2}^{-1}$ contributions are indicated by dashed curves.

The selectivity for high-spin, particle-hole states made from nodeless neutron and $\Lambda$ orbitals is much enhanced, over expectations from plane-wave calculations, by distortion. As one would intuitively expect, transitions to the $1 s_{\Lambda}$ orbit, concentrated in the nuclear interior, are more strongly suppressed by the effects of distortion than transitions to the loosely bound high $l_{\Lambda}$ orbits. To demonstrate these effects, we compare in Table 1 the results of DWBA calculations with full distortion $\left(\pi^{+}\right.$and $\left.\mathrm{K}^{+}\right), \mathrm{K}^{+}$distortion only, $\mathrm{K}^{+}$ Coulomb waves and plane wave (PW) for selected $g_{9 / 2} \rightarrow l_{\Lambda}$ transitions. As can be seen, the effects of distortion are more severe for $\Delta J(=\Delta L)$ less than the maximum value, for transitions involving noded orbits and for larger angles (higher momentum transfer).

With the advent of CEBAF, it will become possible to produce hypernuclei using electron beams. Although $\left(e, e^{\prime} \mathrm{K}^{+}\right)$cross sections are predicted ${ }^{7}$ to be smaller by roughly two orders of magnitude than $\left(\pi^{+}, \mathrm{K}^{+}\right)$cross sections, the projected count rates are comparable to the rates achieved in $\left(\pi^{+}, \mathrm{K}^{+}\right)$experiments at the Brookhaven AGS. For the purpose of investigating the $\Lambda$ mean field in heavy hypernuclei, the $\left(\pi^{+}, \mathrm{K}^{+}\right)$and $\left(e, e^{\prime} \mathrm{K}^{+}\right)$reactions 
are similar with regard to momentum transfer and to nuclear structure factors, despite the fact that spin-flip strength is dominant for $\left(e, e^{\prime} \mathrm{K}^{+}\right)$and small for $\left(\pi^{+}, \mathrm{K}^{+}\right)$. The $\left(e, e^{\prime} \mathrm{K}^{+}\right)$ reaction has some advantages for studying deeply-bound $\Lambda$ orbits since the strong effects of $\pi^{+}$distortion on $\left(\pi^{+}, \mathrm{K}^{+}\right)$cross sections for interior and noded orbits are absent. Apart from an overall scaling factor to account for the different elementary cross section, we expect the results for $\mathrm{K}^{+}$distortion only in Table 1 to give a reliable guide for the $\left(\gamma, \mathrm{K}^{+}\right)$reaction (on a $Z=50$ nucleus). This is because, to a good approximation, the structure factors factorize out of the DWBA expression and, when summed over $\Lambda$ spin-orbit partners (small spin-orbit splitting), are very similar for the high-spin case of $\Delta L=l_{N}+l_{\Lambda}$ with $\Delta S=0$ or $\Delta S=1(\Delta J=\Delta L$ or $\Delta L+1)$. Lower multipoles will contribute relatively more strongly to $\left(\gamma, \mathrm{K}^{+}\right)$than to $\left(\pi^{+}, \mathrm{K}^{+}\right)$cross sections, and the peaks in a theoretical spectrum analogous to that shown in Fig. 1 should fall off less rapidly with increasing $\Lambda$ binding energy.

The most important requirement for improvements in hypernuclear spectroscopy is better energy resolution. Here, an $\left(e, e^{\prime} \mathrm{K}^{+}\right)$program at CEBAF should have a significant advantage over the existing $\left(\pi^{+}, K^{+}\right)$facilities at BNL and KEK, where the energy resolution is about $3 \mathrm{MeV}$. An improved understanding of the elementary $\gamma p \rightarrow \mathrm{K}^{+} \Lambda$ reaction ${ }^{7,8}$ is necessary to make the most of the $\left(e, e^{\prime} \mathrm{K}^{+}\right)$reaction as a tool for probing hypernuclear structure. To this end, the program of first-round experiments at CEBAF includes the measurement of elementary differential cross sections for the photoproduction of $\Lambda$ and $\Sigma^{0}$ hyperons and the polarizat ion of the $\Lambda$ from threshold to about $1.8 \mathrm{GeV}$. Much theoretical work will be required to achieve a satisfactory description of the elementary interaction and to apply-it to the $\left(e, e^{\prime} \mathrm{K}^{+}\right)$reaction on nuclei; ideally, for a full interpretation, elastic $\mathrm{K}^{+}$ scattering data needs to be taken elsewhere at the appropriate energies (elastic scaitering data is also needed to better understand $\left(\mathrm{K}^{-}, \pi^{-}\right)$and $\left(\pi^{+}, \mathrm{K}^{+}\right)$cross sections).

For light $\Lambda$ hypernuclei, some excited state energies and cross sections are available from $\left(\mathrm{K}^{-}, \pi^{-}\right)$and $\left(\pi^{+}, \mathrm{K}^{+}\right)$reactions, together with a few precise measurements of excitation energies from $\left(\mathrm{K}^{--}, \pi^{-} \gamma\right)$ experiments, but these have not yet yielded a unique determination of the various spin-spin, spin-orbit and tensor matrix elements of the $\Lambda \mathrm{N}$ force. Ideally, measurements of $s_{\Lambda}$ doublet splittings are required. The largest doublet splittings are predicted for cases in which the spin-spin component of the $\Lambda \mathrm{N}$ interaction dominates. For example, the gs doublet in ${ }_{\Lambda}^{7} \mathrm{Li}$, where the states have $\mathrm{L}=0$ with $\mathrm{S}=1 / 2$ or $\mathrm{S}=3 / 2$, is predicted ${ }^{6}$ to be split by $\sim 600 \mathrm{keV}$ or by $\sim 440 \mathrm{keV}$ for a set of interaction parameters ${ }^{9}$ which takes into account a failure ${ }^{10}$ to observe a $\gamma-\mathrm{ra}$, transition between the members of the gs doublet in ${ }_{\Lambda}^{10} \mathrm{~B}$. Corresponding splittings for the gs doublet in ${ }_{\Lambda}^{9} \mathrm{Li}$, accessible via the $\left(e, e^{\prime} \mathrm{K}^{+}\right)$reaction on ${ }^{9} \mathrm{Be}$, are $510 \mathrm{keV}$ and $280 \mathrm{keV}$. Thus, even a resolution of a few hundred $\mathrm{keV}$ for the $\left(e, e^{\prime} \mathrm{K}^{+}\right)$reaction may be insufficient to resolve states belonging to all but a few, if any, $s_{\Lambda}$ doublets in light hypernuclei. Nevertheless, high resolution, combined with the access via spin-flip amplitudes to states weakly populated in $\left(\mathrm{K}^{-}, \pi^{-}\right)$and $\left(\pi^{+}, \mathrm{K}^{+}\right)$reactions, could lead to much improved spectroscopic studies. However, it seems unlikely that studies involving further coincidences with hypernuclear $\gamma$-rays or weak-decay products will be possible, at least in the near term. 
In the meantime, an ambitious program of hypernuclear studies is being pursued at KEK, with new superconducting spectrometers being readied ${ }^{11}$ for $\left(\pi^{+}, K^{+}\right)$and stopped $\left(\mathrm{K}^{-}, \pi\right)$ experiments to begin in 1990 . Emphasis is being placed on an ability to perform coincidence experiments involving, in particular, weak-decay products from both the mesonic and non-mesonic decay modes of hypernuclei. If significant polarization of a hypernucleus is achieved in the $\left(\pi^{+}, \mathrm{K}^{+}\right)$reaction through the combined effects of spin-flip amplitudes and distortion, as is predicted, ${ }^{12}$ measurements of angular correlations of decay products will also be possible.

Clearly, more progress will be possible with hadronic probes of hypernuclei if nore intense beams and better resolution become available. For example, beams with nearly two orders of magnitude higher $\pi^{+}$intensity than are available at BNL or KEK, with a resolution of $200 \mathrm{keV}$ for the $\left(\pi^{+}, \mathrm{K}^{+}\right)$reaction, are being considered at LAMPF as part of the PILAC proposal and intense secondary beams could become available at KAON. The combination of good resolution with the ability to utilize the $\left(\pi^{+}, \mathrm{K}^{+} \gamma\right)$ or the $\left(\mathrm{K}^{-}, \pi^{-} \gamma\right)$ reaction, already demonstrated at Brookhaven, would open the door to precise spectroscopic studies. A more comprehensive discussion of, and list of references relating to, $\Lambda$-hypernuclear structure is given in a recent review on the electroproduction of hypernuclei ${ }^{13}$.

\section{ACKNOWLEDGEMENTS.}

I would like to thank C. B. Dover and A. Gal for their collaboration on many aspects of the work reported here.

\section{REFERENCES}

1. R. E. Chrien et al, Nucl. Phys. A478 (1988) 705c.

2. D. J. Millener, A. Gal and C. B. Dover, Phys. Rev. C38 (1988) 2700.

3. Y.Yamamoto, H. Bandō and J. Žofka, Prog. Theor. Phys. $\underline{80}$ (1988) 757.

4. T. Motoba, H. Bandō, R. Wünsch and J. Žofka, Phys. Rev. C38 (1988) 1322.

5. J. Kasagi, G. M. Crawley, E. Kashy, J. Duffy, S. Gales, E. Gerlic and D. Friesel, Phys. Rev. C28 (1983) 1065.

6. D. J. Millener, A. Gal, C. B. Dover and R. H. Dalitz, Phys. Rev. C31 (1985) 499.

7. C. Bennhold and L. E. Wright, Phys. Rev. C39 (1989) 927; A. S. Rosenthal, D. Halderson, K. Hodgkinson and F. Tabakin, Ann. Phys. (N.Y.) 184 (1988) 33.

8. R. A. Adelseck and B. Saghai, in press, Phys. Rev. C,42 (1990), in press.

9. V. N. Fetisov, L. Majling, J. Z̆ofka and R. A. Eramzhyan, preprint (Institute of Nuclear Research, Moscow).

10. R. E. Chrien et al., Phys. Rev. C41 (1989) 1062.

11. 1988 Int. Symp. on Hypernuclear and Low-Energy Kaon Physics, Padova, Italy, eds. T. Bressani, F. Cannata, J. Lowe and R. A. Ricci, Il Nuovo Cimento 102A (1989) 1.

12. H. Bandō, T. Motoba, M. Sotona and J. Žofka, Phys. Rev. C39 (1989) 587; T. Kishimoto, H. Ejiri and H. Bandō, Phys. Lett. 232B (1989) 24.

13. C. B. Dover and D. J. Millener, Electroproduction of Strangeness, in: Modern Topics in Electron Scattering, eds. B. Frois and I. Sick (World Scientific, Singapore), in press. 


\section{DISCLAIMER}

This report was prepared as un account of work sponsored by an agency of the United States Government. Neither the United States Government nor any agency thereof, nor any of their employees, makes any warranty, express or implied, or assumes any legal liability or responsibility for the accuracy, completeness, or usefulness of any information, apparatus, product, or process disclosed, or represents that its use would not infringe privately owned rights. Reference herein to aily specific commercial product, process, or service by trade name, trademark, manufacturer, or otherwise does not necessarily constitute or imply its endorsement, recommendation, or favoring by the linited States Government or any agency thereof. The views and opinions of authors expressed herein do not necessarily state or reflect those of the United States Government or any agency there of. 

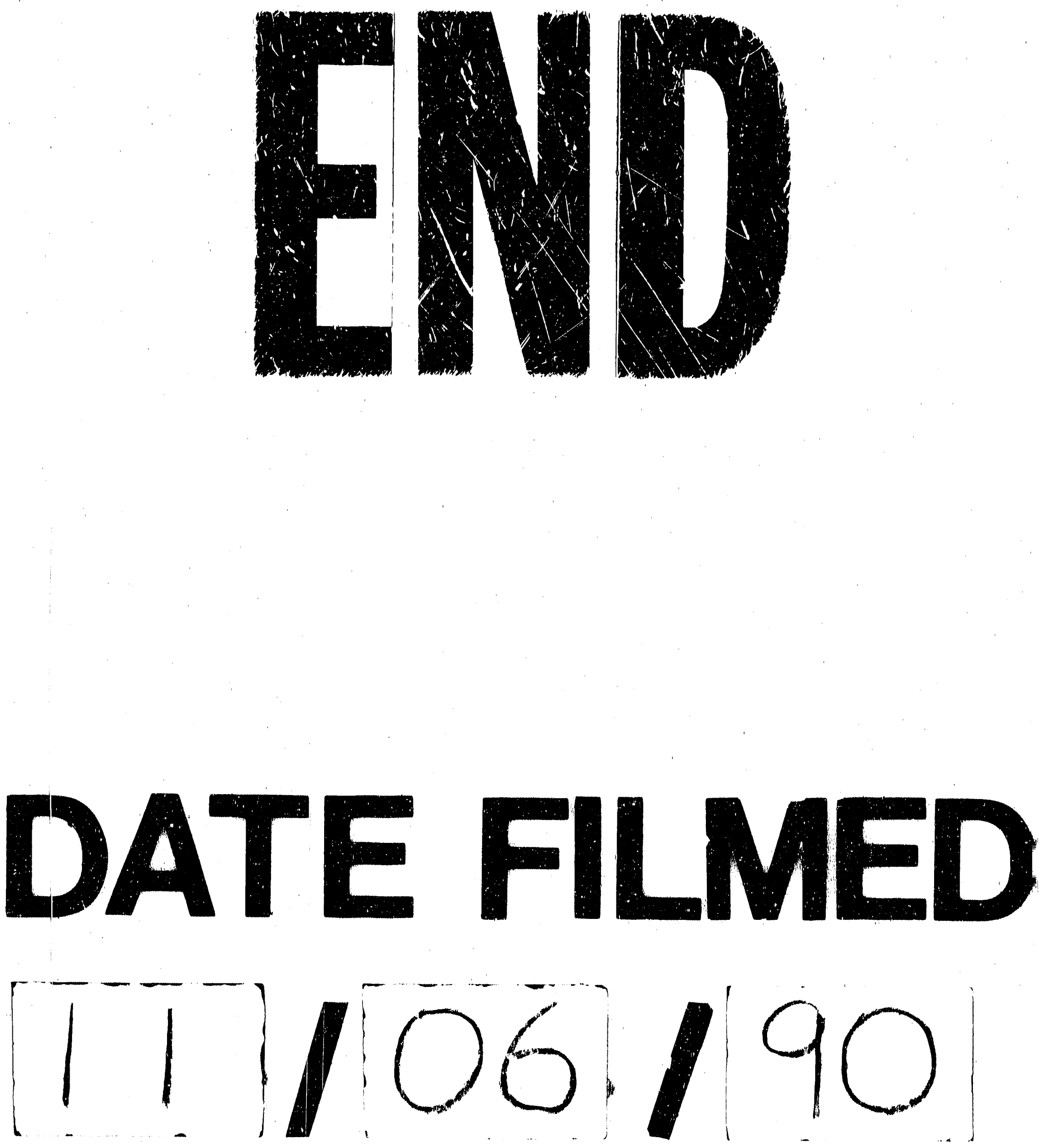
$\mid$ 